\title{
The process by which perceived autonomy support predicts motivation, intention, and behavior for seasonal influenza prevention in Hong Kong older adults
}

Pak-Kwong Chung ${ }^{1 *}$, Chun-Qing Zhang ${ }^{1}$, Jing-Dong Liu', Derwin King-Chung Chan², Gangyan $\mathrm{Si}^{3}$ and Martin S. Hagger ${ }^{1,4,5}$

\begin{abstract}
Background: This study examined the effectiveness of a theoretical framework that integrates self-determination theory (SDT) and the theory of planned behavior (TPB) in explaining the use of facemasks to prevent seasonal influenza among Hong Kong older adults.

Methods: Data were collected at two time points in the winter in Hong Kong, during which influenza is most prevalent. At Time 1, older adults $(N=141)$ completed self-report measures of SDT (perceived autonomy support from senior center staff, autonomous motivation for influenza prevention) and TPB (attitude, subjective norm, perceived behavioral control, and intention for influenza prevention) constructs with respect to facemask used to prevent infection. Two weeks later, at Time 2, participants' acceptance of a facemask to prevent influenza in the presence of an experimenter with flu-like symptoms was recorded.

Results: Path analysis found that perceived autonomy support of senior center staff was positively and significantly linked to autonomous motivation for facemask use, which, in turn, was positively related to intentions to wear facemasks through the mediation of attitude, subjective norm, and perceived behavioral control. However, the effect of intention on facemask use was not significant.

Conclusions: Results generally support the proposed framework and the findings of previous studies with respect to intention, but the non-significant intention-behavior relationship may warrant future research to examine the reasons for older adults not to wear facemasks to prevent seasonal influenza despite having positive intentions to do so.
\end{abstract}

Keywords: Elderly, Facemask wearing, Infection, Infectious diseases, Self-determination theory, Theory of planned behavior

\section{Background}

Seasonal influenza is an acute epidemic of the influenza virus that quickly and easily spreads from person to person [1]. Annual seasonal epidemics peak during the winter months in temperate regions such as Hong Kong. Epidemics cause mild-to-severe illness and can be fatal in vulnerable groups such as the elderly [2]. Everyday preventive actions, such as facemask wearing, play an important role

\footnotetext{
* Correspondence: pkchung@hkbu.edu.hk

'Department of Physical Education, Hong Kong Baptist University, 224

Waterloo Road, Kowloon Tong, Kowloon, Hong Kong, Special Administrative Region of China

Full list of author information is available at the end of the article
}

in the prevention seasonal influenza epidemics $[3,4]$. This is because the main route of human-to-human transmission of the influenza virus is via respiratory droplets when an individual is close contact with someone who has the influenza virus and displays influenza symptoms [5]. In community settings, wearing a facemask is an effective means to minimize transmission of influenza, particularly in areas of high population density. In order to promote the adoption of behaviors like facemask use to prevent seasonal influenza infection, previous research has demonstrated that psychological factors such as motives and intentions are likely play a key role $[6,7]$. 
Researchers have attempted to predict and understand health behavior using behavioral theories and models adopted from social psychology [8]. Among the models that have been applied to understand influenza prevention behaviors, most have adopted a social-cognitive approach, in which preventive behaviors such as vaccination and facemask wearing are viewed as intentional behaviors based on beliefs and expectancies $[6,9,10]$. Prominent among these social cognitive approaches is the theory of planned behavior (TPB) [11]. According to the theory, intention, defined as the extent to which individuals will invest effort in pursuit of an action, is the most proximal predictor of behavior. Intention is the function of attitude (i.e., personal evaluation of how good, useful, valuable, and pleasant the behavior is), subjective norm (i.e., beliefs that the behavior is consistent with the expectations of significant others), and perceived behavioral control (i.e., beliefs in the availability of sufficient personal resources, in relation to barriers or risk factors, to execute the behavior). The TPB has been widely applied and tested in health-related behavioral contexts with meta-analytic studies supporting its effectiveness in predicting various health behaviors $[12,13]$. The predictive efficacy of the TPB with respect to influenza prevention has also been supported in research on influenza vaccination behaviors $[14,15]$.

Apart from the TPB, another important theory in social psychology that has been frequently applied in health contexts is self-determination theory (SDT) $[16,17]$. SDT makes the distinction between different forms of motivation based on its quality rather than quantity: autonomous motivation, controlled motivation, and amotivation. Autonomous motivation is the most self-determined form of motivation. Autonomously motivated individuals perform behaviors for the intrinsic value of the behavior, or to attain personally-important values or goals that represent their true sense of self. In contrast, individuals experiencing actions as controlled motivated engage in behaviors due to external pressures or externally-referenced obligations. Amotivation represents a lack of motivation such that individuals do not know why they engage in their actions at all. Individuals experiencing actions as controlled motivated will only perform an action when the external demands or controlling contingencies are present and the actions will cease once the contingencies are no longer present. Of the three types of motivation identified in SDT, autonomous motivation is proposed to be more favorable to behavioral persistence and well-being because the individual is motivated for self-referenced reasons and in the absence of external reinforcement or contingency [17].

Consistent with the need to promote autonomous motivation toward health behaviors, according to the SDT, creating a context or 'environment' that cultivates autonomous motivation is an effective means to promote autonomous motivation and change behavior $[18,19]$. In an influenza prevention context, interventionists may capitalize on autonomy support techniques as a means to promote autonomous motivation [20]. For communitydwelling older adults, their senior center serves as an important vehicle to present autonomy-supportive messages to promote behaviors that could enhance health and wellbeing, including preventive behaviors to reduce the transmission of potentially life-threatening infections [21].

Recent approaches have attempted to integrate concepts from the TPB and SDT in a unified model to explain health behavior. The integrated model proposes that the motivational variables from SDT are distal predictors of the social cognitive variables from TPB, which are, in turn, considered the proximal antecedents of action $[22,23]$. The rationale for the integration arises from a key tenet of SDT that individuals who perceive their reasons for acting autonomously should view their behavior as one they should approach. In order to enact the behavior, the individual should align his or her beliefs regarding future engagement in the behavior to be consistent with their motives (i.e., to form positive beliefs about the behavior and form intentions to engage in it). Promoting autonomous motivation, therefore, may be a means to promote behavioral engagement through the enhancement of positive beliefs regarding the behavior and the formation of intentions to engage in the behavior in the future. The model has been tested in many health contexts including physical activity, binge drinking, injury management, sugar consumption, and myopia prevention [24-29].

Although a recent study also applied the integrated model to predict behavior in the context of H1N1 pandemic, participants were presented with a hypothetical situation and were not actually facing an actual pandemic and no actual facemask wearing behavior was assessed. [20]. Even though intention is theoretically the most proximal predictor of behavior, there has been growing number of concerns about the differential predictive power of intention on health behavior, and the so-called intention-behavior gap has raised concerns over the adequacy of intentions in explaining future behavior [30-32]. It is, therefore, important that test of the integrated model include prospective measures of actual behavior, particularly in influenza prevention contexts.

The purpose of the current study was to examine the efficacy of an integrated model based on SDT and TPB to predict facemask wearing to prevent seasonal influenza in a sample of Hong Kong older adults. We adopted a two-week prospective correlational design during Hong Kong's winter, the peak season for seasonal influenza, and we included an actual measure of the target behavior. Specifically, we measured the motivational and social cognitive variables from the integrated model at Time 1 (baseline) and followed that up two-weeks 
later at Time 2 with an assessment of actual facemaskwearing behavior. Building on the integrated model, it was hypothesized that: (1) Perceived autonomy support from senior center staff for facemask wearing would be positively and directly related to autonomous motivation (hypothesis 1a) and positively and indirectly related to attitude, subjective norm, and perceived behavioral control via the mediation of autonomous motivation (hypothesis 1b); (2) Autonomous motivation would be positively and directly related to attitude, subjective norm, and perceived behavioral control (hypothesis 2a) and positively and indirectly related to intention via the mediation of attitude, subjective norm, and perceived behavioral control (hypothesis 2b); (3) Attitude, subjective norm, and perceived behavioral control would be positively and directly associated with intention (hypothesis 3a) and positively and indirectly related to actual facemask wearing behavior via the mediation of intention (hypothesis $3 \mathrm{~b}$ ).

\section{Method \\ Participants}

Ethical clearance was granted by the committee of Research Ethics and Safety (HASC) at Hong Kong Baptist University. We obtained approval and on-site assistance to recruit participants from one senior center in Hong Kong using convenience sampling. This center has over 1500 registered Chinese-speaking community-dwelling Hong Kong older adults aged 60 years and older. A total of 180 older adults expressed an interest in participating to senior center staff and members of the research team. Of these eligible participants, 141 agreed to participate and signed consent forms (response rate $=78.33 \%$ ). The sample comprised of 14 males and 127 females ( $M$ age $=75.23, S D=6.52$, range 60 to 85 ) participated the study at Time 1 . At Time 2, 137 participants (13 males, 124 females; $\left.M_{\text {age }}=75.10, S D=6.49\right)$ remained in the study (retention rate $=97.16 \%$ ).

\section{Design and procedure}

Data were collected at two time points with a two-week interval in the November of 2014. At Time 1, during the peak season of winter influenza in Hong Kong, participants completed survey measures of study variables under the supervision of researchers following an introductory session in which the purpose and procedure of the study was explained and written informed consent obtained. The surveys took 10 to $15 \mathrm{~min}$ to complete. Senior center staff were not present during the completion and were not able to see participants' responses. At Time 2, participants were invited to a face-to-face interview in a private room to ostensibly assess their knowledge of H1N1. The true purpose of the interview was to assess participants' facemask wearing behavior for influenza prevention. During the course of the interview, the interviewer wore a facemask and feigned influenzalike symptoms (e.g., coughing, sneezing). Before the start of the interview, the interviewer told the participants in an offhand manner that he/she had caught "the "flu", and that surgical facemasks were available at the desk that they could take and use for free. This allowed the interviewers to record participants' facemask-wearing behavior. On completion of the interview, participants were debriefed regarding the cover story and informed that the interviewer had feigned influenza symptoms and worn the facemask as a prop to model the behavior.

\section{Measures}

\section{Perceived autonomy support}

Perceived autonomy support from staff of senior center was measured using the six-item Health Care Climate Questionnaire (HCCQ) [18]. Participants were provided with a common stem: "When the staff at the senior center asks me to wear a facemask in an enclosed public place..." followed by the six items (e.g., "I feel that he/ she has provided me choices and options"). Responses were made on a 7-point scale anchored by 1 ("not at all true") and 7 ("very true"). The Chinese version of the HCCQ has demonstrated sufficient validity and reliability [20].

\section{Autonomous motivation}

The six-item autonomous motivation subscale from the Treatment Self-Regulation Questionnaire (TSRQ) [33] was used to measure autonomous motivation. The stem of TSRQ items was modified to refer the specific behavior of interest (i.e., "I want to wear a facemask in an enclosed public place because ...”). Participants responded to items on 7-point Likert scales with 1 ("not at all true") and7 ("very true") as scale anchors. The Chinese version of TSRQ has demonstrated sufficient validity and reliability [20].

\section{Theory of planned behavior}

Measure of the TPB variables were based on Ajzen's guidelines [34]. Measures of subjective norm (3 items; e.g., "It is expected of me to wear facemask in an enclosed public place in the forthcoming month."), perceived behavioral control (5 items; e.g., "It is possible for me to wear facemask in an enclosed public place in the forthcoming month."), and intention (3 items; e.g., "I intend to wear facemask in an enclosed public place in the forthcoming month.") were rated on 7-point Likert scales with 1 ("strongly disagree") and 7 ("strongly agree") as anchors. Attitudes were measured using 7 items preceded by a common stem, "For me to wear facemask in an enclosed public place in the forthcoming month would be ..." followed by a series of 7-point 
semantic differential scales: extremely harmful-extremely beneficial, extremely unpleasant-extremely pleasant, extremely worthless-extremely valuable, extremely badextremely good, and extremely unenjoyable-extremely enjoyable.

\section{Facemask-wearing behavior}

Facemask-wearing behavior was assessed at Time 2 during the face-to-face interview. The interviewer disclosed in an offhand manner that they had caught influenza and feigned influenza symptoms. Participants were made fully aware of the availability of facemasks and that they were free to use. The interviewer was trained to observe and record the correct use of the facemasks according to World Health Organization guidelines (i.e., complete coverage of mouth and nose) [5]. The interviewers recorded a 1 ("yes") when participants took and used the facemasks correctly and otherwise recorded a 0 ("no").

\section{Control variables}

Control variables included past facemask-wearing habit (i.e., whether or not they had worn facemasks previously; 1 item), knowledge of the benefits of facemask wearing (i.e., knowing that facemask wearing can prevent influenza; 1 item), frequency of influenza infection during the past 6 months (1 item), and perceived susceptibility. Perceived susceptibility (e.g., "I have an increased risk of falling ill with influenza"; 3 items) was rated on a 7 point Likert scale with 1 ("strongly disagree") and 7 ("strongly agree") as scale anchors [7].

\section{Data analysis}

Given that the Time 2 facemask wearing behavior was a categorical variable (yes/no), the proposed integrative model (see Fig. 1) was tested using path analysis (i.e., observed variables) with a variance-adjusted weighted-least squares (WLSMV) estimation method using the Mplus 7.3 [35]. Variables including facemask wearing habit, knowledge of facemask wearing benefits, frequency of influenza during the past 6 months, and perceived susceptibility were included as control variables. Adequacy of the fit of the proposed model with the data was based on multiple criteria for assessing goodness of fit including the comparative fit index (CFI), the root-mean-square error of approximation (RMSEA) and the weighted root mean square residual (WRMSR). Values exceeding .90 for the CFI and less than .08 and 1.00 for the RMSEA and WRMSR [36, 37], respectively, indicate good fit. For our tests of mediated effects, mediation was confirmed when the indirect effect of a predictor variable (e.g., autonomous motivation) on an outcome variable (e.g., intention) via a mediator (e.g., attitude) was statistically significant and the confidence interval of the effect size did not include zero.

\section{Results}

\section{Preliminary analysis}

In terms of the facemask wearing habit, most of the participants reported wearing facemasks previously $(n=124)$, while a small number of them reported that they did not been used to wearing facemasks previously $(n=17)$. With respect to knowledge of the

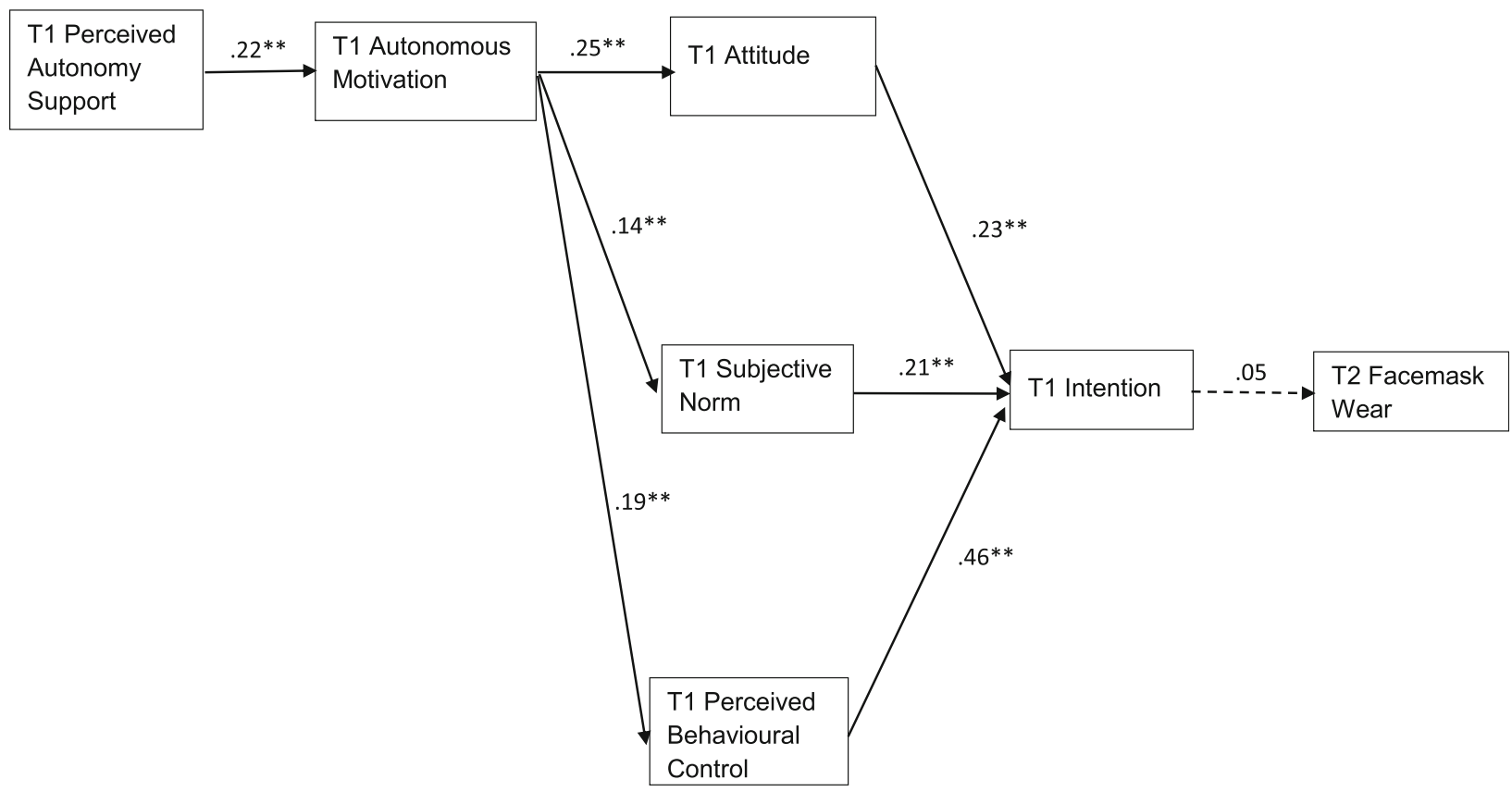

Fig. 1 Path Estimates of the Integrated Model. For clarity reasons, the statistically non-significant paths are not displayed. ${ }^{* *} p<.01 ;{ }^{*} p<.05$ 
benefits of facemask wearing, most of the participants reported knowing facemask wearing can prevent flu $(n=132)$, while a small number reported they did not know facemask wearing can prevent flu $(n=9)$. With regards to the frequency of influenza during the past 6 months, most of the participants reported that they had not caught influenza $(n=120)$. In comparison, only 11 participants reported that they caught influenza once, seven participants reported they had had the flu twice, and only three participants reported catching influenza three times. Missing data analysis revealed no significant pattern (missing data $=0.18 \%$ ) and the small number if missing cases was replaced using mean substitution. Descriptive statistics and intercorrelations of the study variables are presented in Table 1.

\section{Path analysis}

The model exhibited adequate fit with the data, $x^{2}$ (3) $=1.58, p=.664, \mathrm{CFI}=1.00$, WRMR $=.178$, RMSEA $(90 \% C I)=.000(.000, .112)$. Direct, indirect, and total effects of the path estimates of the integrated model are presented in Table 2 and Fig. 1. Effects of the control variables were not significant except the effect of frequency of influenza over the previous six months on autonomous motivation $(\beta=-.18,95 \% C I$ [ -.344 to -.024$]$, $p=.025)$, and they are not, therefore, displayed in Fig. 1.

Focusing on the effects encompassed by hypothesis 1, we observed a statistically significant and positive effect of perceived autonomy support on autonomous motivation consistent with our hypothesis (hypothesis 1a). The indirect effects of perceived autonomy support on attitude and subjective norm were statistically significant as hypothesized (hypothesis $1 \mathrm{~b}$ ), although the direct effects were not. As predicted, the total, direct, and indirect effects of perceived autonomy support on perceived behavioral control were also statistically significant (hypothesis $1 \mathrm{~b})$.

Statistically significant and positive associations were observed for autonomous motivation on attitude, subjective norm, and perceived behavioral control, as hypothesized (hypothesis 2a). The indirect effects of autonomous motivation on intention mediated by attitude, subjective norm, and perceived behavior control were all statistically significant, consistent with predictions (hypothesis $2 \mathrm{~b}$ ).

We found statistically significant and positive effects of attitude, subjective norm, and perceived behavioral control on intention, as predicted (hypothesis 3a). However, contrary to predictions, the effect of intention on our measure of facemask wearing was small and not statistically significant (hypothesis $3 \mathrm{~b}$ ).

\section{Discussion}

Building on an integrated model of SDT and TPB [22, 23, 27], we tested effects among perceived autonomy support, autonomous motivation, attitudes, subjective norms, perceived behavioral control, intention, and behavior for facemask wearing during peak influenza season among elderly people in Hong Kong. Consistent with our hypotheses, we found statistically significant and positive effects of (1) perceived autonomy support on autonomous motivation, attitude, subjective norm, and perceived behavior control; (2) autonomous motivation on attitude, subjective norm, and perceived behavioral control, and intention; and (3) attitude, subjective norm, and perceived behavioral control, on intention. There were also significant and positive indirect effects of perceived autonomy support on attitude, subjective norm, and perceived behavior control via autonomous motivation, and of autonomous motivation on intentions via attitude, subjective norm, and perceived behavior control. However, we found no effect of intention on our measure of actual facemask wearing, and, consequently, no indirect effects of the motivational and social-cognitive variables on behavior mediated by intention. Our findings, therefore, suggest that the motivational and social-cognitive constructs outlined in the integrated model are effective in predicting intentions to wear a facemask during the peak winter season, but, critically, intentions were not predictive of individuals' actual engagement in facemask wearing behavior.

Table 1 Summary of correlations, means, standard deviations (SD), and internal consistencies of the self-reported questionnaires

\begin{tabular}{|c|c|c|c|c|c|c|c|c|c|c|}
\hline & & Range & Mean & SD & $a$ & 1 & 2 & 3 & 4 & 5 \\
\hline 1 & Perceived autonomy support & $6-42$ & 33.36 & 4.27 & .77 & & & & & \\
\hline 2 & Autonomous motivation & $6-42$ & 36.02 & 2.45 & .67 & $.26^{* *}$ & & & & \\
\hline 3 & Attitude & $7-49$ & 38.63 & 5.97 & .68 & $.25^{* *}$ & $.33^{* *}$ & & & \\
\hline 4 & Subjective norm & $3-21$ & 16.55 & 3.10 & .79 & $.17^{*}$ & $.18^{* *}$ & $.34^{* *}$ & & \\
\hline 5 & Perceived behavioral control & $5-35$ & 29.94 & 2.97 & .90 & $.28^{* *}$ & $.24^{* *}$ & $.40^{* *}$ & $.23^{* *}$ & \\
\hline 6 & Intention & $3-21$ & 16.56 & 3.30 & .88 & .11 & $.22^{*}$ & $.45^{* *}$ & $.39^{* *}$ & $.57^{* *}$ \\
\hline
\end{tabular}

${ }^{* *} p<.01$ at two-tailed

${ }^{*} p<.05$ at two-tailed 
Table 2 Total, direct, and indirect effects of the proposed paths in the Integrated Model

\begin{tabular}{|c|c|c|c|}
\hline & Effect size & Confidence interval & $p$ value \\
\hline T1 Perceived support $\longrightarrow$ T1 Autonomous motivation & $.22^{* *}$ & .055 to .384 & .009 \\
\hline \multicolumn{4}{|l|}{ T1 Perceived support $\longrightarrow$ T1 Attitude } \\
\hline Total effect & $.19^{*}$ & .005 to .380 & .044 \\
\hline Direct effect & .14 & -.045 to .320 & .140 \\
\hline Indirect effect via Autonomous motivation & $.06^{*}$ & .005 to .105 & .032 \\
\hline \multicolumn{4}{|l|}{ T1 Perceived support $\longrightarrow$ T1 Subjective norm } \\
\hline Total effect & .13 & -.025 to .288 & .099 \\
\hline Direct effect & .10 & -.055 to .256 & .205 \\
\hline Indirect effect via Autonomous motivation & $.03^{*}$ & .001 to .056 & .042 \\
\hline \multicolumn{4}{|l|}{ T1 Perceived support $\longrightarrow$ T1 PBC } \\
\hline Total effect & $.23^{* *}$ & .058 to .407 & .009 \\
\hline Direct effect & $.19^{*}$ & .024 to .356 & .025 \\
\hline Indirect effect via Autonomous motivation & $.04^{*}$ & .006 to .079 & .022 \\
\hline T1 Autonomous motivation $\longrightarrow$ T1 Attitude & $.25^{\star *}$ & .120 to .380 & $<.001$ \\
\hline T1 Autonomous motivation $\longrightarrow$ T1 Subjective norm & $.14^{\star *}$ & .043 to .238 & .005 \\
\hline $\mathrm{T} 1$ Autonomous motivation $\longrightarrow \mathrm{T} 1 \mathrm{PBC}$ & $.19^{\star \star}$ & .087 to .300 & $<.001$ \\
\hline \multicolumn{4}{|l|}{ T1 Autonomous motivation $\longrightarrow$ T1 Intention } \\
\hline Total effect & $.16^{\star}$ & .007 to .313 & .040 \\
\hline Direct effect & -.02 & -.156 to .127 & .839 \\
\hline Total indirect effects & $.18^{* *}$ & .099 to .251 & $<.001$ \\
\hline Indirect effect via Attitude & $.06^{*}$ & .010 to .103 & .016 \\
\hline Indirect effect via Subjective norm & $.03^{*}$ & .003 to .056 & .027 \\
\hline Indirect effect via PBC & $.19^{\star *}$ & .024 to .152 & .007 \\
\hline T1 Attitude $\longrightarrow \mathrm{T} 1$ Intention & $.23^{\star *}$ & .086 to .366 & .002 \\
\hline T1 Subjective norm $\longrightarrow$ T1 Intention & $.21^{\star *}$ & .131 to .294 & $<.001$ \\
\hline T1 PBC $\longrightarrow$ T1 Intention & $.46^{\star *}$ & .345 to .568 & $<.001$ \\
\hline T1 Intention $\longrightarrow$ T2 Facemask wearing & .05 & -.213 to .317 & .703 \\
\hline
\end{tabular}

\section{Efficacy of the integrated model and its component theories}

Current findings are consistent with the hypotheses of the integrated model and its component theories when it comes to predicting intentions. Perceived autonomy support was related to autonomous motivation for adopting health-related behaviors consistent with the predictions of SDT $[16,17,38]$, a key component theory of the integrated model $[22,23]$. The finding is also consistent with the findings of a previous study on facemask wearing [20]. The results suggest that establishing an autonomy supportive environment or 'motivational climate' toward influenza prevention behaviors such as facemask wearing will be effective in fostering autonomous motivation toward the behavior.

Consistent with previous studies of influenza prevention behaviors such as facemask wearing and vaccination using the TPB $[14,15,20]$, attitudes, subjective norms, and perceived behavior control had positive, significant, and medium-to-large associations with the intentions of wearing facemasks in Hong Kong older adults. Among these three variables, perceived behavioral control had the strongest effects relative to attitudes and subjective norms. It was also the most prominent variable in the transmission of indirect effects of autonomous motivation on intention. Given that the TPB is, by and large, an integration of the personal (i.e., attitude) and social (i.e., subjective norm) antecedents of intention from the reasoned action approach $[39,40]$, and the self-efficacy related component from social cognitive theory [41], the increase of older adults' personal resources (e.g., selfefficacy) and the decrease of the hindrance from barriers (e.g., easy access to facemasks when needed) seem to be most efficacious in accounting for variance in intentions 
to engage in the focal health behavior. Current findings provide formative evidence to support interventions to facilitate greater confidence in wearing facemasks and to overcome barriers to doing so.

The significant and positive direct effects of perceived autonomy support on attitude and perceived behavioral control indicated that a supportive motivational climate may affect the belief-based TPB variables. This is in line with previous studies [20, 24, 25, 27] and it further corroborates the integrated model $[22,23]$. That is, autonomous motivation from SDT underpins the belief-based social cognitive variables of TPB, and the current study extends this to facemask wearing to prevent influenza transmission. The prominent role of control-related beliefs for this health behavior, and its mediating role in translating autonomous motives into intentions, is consistent with other theories which feature self-efficacy as a prominent component [42]. It is also consistent with previous research that has adopted the integrated model and demonstrated that perceived behavioral control is a key predictor and mediating factor of the influence of autonomous motivation on intentions [43].

\section{Intentions do not predict behavior}

The intentions of facemask wearing alone may be insufficient or unimportant when it comes to engaging in this particular behavior. In a post hoc analysis, we found that the majority of participants (89.05\%) scored above the mid-point on the intention scale but only two fifths of them chose to wear the face masks (39.40\%) in our scenario. Considering these data, it would be reasonable to assume that the majority of participants should be considered inclined abstainers or unsuccessful intenders [30, 44], or have weak or unstable [45-47] intentions. Given that older adults' facemask wearing habits, knowledge of whether wearing facemasks can prevent influenza, frequency of influenza over the previous 6 months, and perceived susceptibility to influenza were controlled for, it is reasonable to conclude that the current findings are consistent with the well-documented intention-behavior gap.

A number of factors may account for this 'gap'. First, it emerged from the qualitative debriefing session conducted after the study had been completed that many of the participants chose not to wear a facemask because the interviewer was wearing a facemask, and they believed that they were, therefore, safe. Others reported that they were put off wearing facemasks themselves because it would make it difficult or uncomfortable to breathe, a notable barrier. As this is anecdotal evidence rather than a formal assessment, it may be important in future research to examine participants' specific beliefs regarding the behavior, rather than global or direct measures of attitudes and perceived behavioral control. Such an approach may identify the influential behavioral (e.g., beliefs that people with illnesses will wear facemasks to reduce risk to others) and control (e.g., wearing masks makes it difficult or uncomfortable to breathe) beliefs that may influence the formation of intentions. Omission the beliefs that precede intentions aside, it may also be important to study the underlying volitional mechanisms from dual-phase models of action like the model of action phases [48] and the Health Action Process Approach [44] that determine the enactment of intentions. Incorporation of volitional components like implementation intentions, action planning, and coping planning into the study of facemask wearing may assist in identifying the possible moderators of the intention behavior relation.

Similarly, it may also be important to look at the implicit, non-conscious or 'automatic' influences on action. Research has indicated that self-reported habit predicts behavior as do implicitly measured attitudes and motives [49-51]. Such influences likely account for action independent of the intentional route to behavior, and therefore determines action beyond an individual's awareness. The likely process involved is that the implicit beliefs are associated with schema, or memory structures, which outline patterns of action which have been well learned and reinforced over time. Such patterns are activated when the implicit belief or attitude is cued, and, as a consequence, an individual's behavior is initiated automatically $[27,52]$. This does not mean that individuals act like 'automatons', blindly carrying out actions. The actual behavior may involve considerable planning and effort, it is just that the behavioral pattern is set in motion in a quick, efficient manner making the behavior subjectively very easy to enact. Implicit beliefs are also more likely to facilitate the enactment of the intended action, or failing to do the action, if it is either rewarding or relatively easy to do. Future research should examine individuals' implicit beliefs toward illness and implicit beliefs toward the behavior, with respect to facemask wearing and assess their contribution to action. This would be consistent with further integrated models that have attempted to incorporate implicit components alongside motivational and volitional components of action in a unified framework [23].

\section{Strengths and limitations}

The current study has numerous strengths including a focus on an important health-related behavior aimed at preventing influenza infection in a vulnerable population and has the potential to save lives, recruitment of a hard-to-reach population of older adults, adoption of an integrated theoretical model that has shown efficacy in predicting health behavior, and the use of valid and reliably instruments and a prospective design, and the use of an externally-verified observational measure of behavior instead of self-reports which have inherent biases. 
Although our findings offer insight into the motivational processes that relate to facemask use in Hong Kong older adults to prevent influenza transmission during peak season, a number of limitations of the study should be identified and discussed. First, participants in the current study were ethnically and geographically homogeneous since they were recruited from the same senior center. All participants were Hong Kong Chinese, and most of them were female. In future, it would be important to replicate the findings in more diverse sample of older adults to provide further evidence on the validity and generalizability of study findings. Secondly, inference of causal relations among variables in the integrated model cannot be made even with the adoption of a prospective design. The causal direction of effects in the model can only be inferred from the theory rather than the data [53], it is, therefore, imperative that researchers also adopt experimental paradigms to manipulate key variables in the nomological network proposed by the theory and observe their effects on outcomes. Finally, the current study did not conduct an a priori statistical power analysis and adopted a manifest rather than latent variable approach given the relatively small sample. Future research should seek to conduct a power analysis beforehand, collect data from a larger sample and use a latent variable modeling approach which would control for measurement error [54].

\section{Conclusion}

In conclusion, findings of the current study provided support for the motivational aspects of the integrated model to the wearing of facemasks to prevent seasonal influenza in a sample Hong Kong older adults. However, our research did not support the link between intentions to wear facemasks and a situated decisional measure of facemask wearing in a real-life context. Further examination of the potential alternative processes and moderators of the link between intentions and behavior in this context may provide insight on contexts in which intentions to wear facemasks lead to actual facemask wearing behavior, and the processes involved.

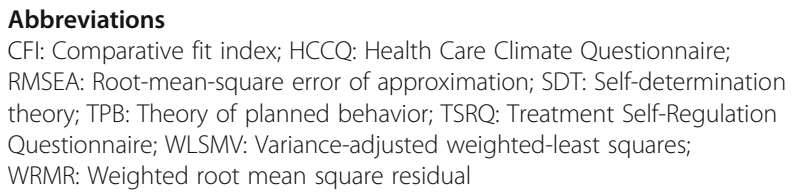

\section{Acknowledgements}

Not applicable.

\section{Funding}

This study was funded by Faculty Research Grant (FRG), Hong Kong Baptist University (grant number FRG1-13-14-066).

\section{Authors' contributions}

All authors were involved in the design of the study protocol. PKC, CQZ, JDL, and DKC were involved in the data acquisition. PKC and CQZ conducted all analyses and wrote the first draft. PKC, CQZ, DKC, and MSH contributed to the interpretation of the results. All authors critically reviewed the manuscript and approved the final version.

\section{Ethics approval and consent to participate}

The current study involves human participants and the research protocol had been approved by the committee of Research Ethics and Safety (HASC) at Hong Kong Baptist University. All participants provided written informed consent to participate.

Consent for publication

Not applicable.

\section{Competing interests}

The authors declare that they have no competing interests.

\section{Publisher's Note}

Springer Nature remains neutral with regard to jurisdictional claims in published maps and institutional affiliations.

\section{Author details}

'Department of Physical Education, Hong Kong Baptist University, 224 Waterloo Road, Kowloon Tong, Kowloon, Hong Kong, Special Administrative Region of China. ${ }^{2}$ Institute of Human Performance, University of Hong Kong, Pokfulam, Hong Kong, Special Administrative Region of China. ${ }^{3}$ Sport Psychology Center, Hong Kong Sports Institute, Hong Kong, China. ${ }^{4}$ Health Psychology and Behavioural Medicine Research Group, School of Psychology and Speech Pathology, Curtin University, Perth, Australia. ${ }^{5}$ Department of Sport Sciences, University of Jyväskylä, Jyväskylä, Finland.

Received: 14 September 2016 Accepted: 19 July 2017

Published online: 28 July 2017

\section{References}

1. World Health Organization. Influenza (Seasonal). 2014. Retrieved from http:// www.who.int/mediacentre/factsheets/ss211/en/.

2. Harper SA, Bradley JS, Englund JA, File TM, Gravenstein S, Hayden FG, et al. Seasonal influenza in adults and children-diagnosis, treatment, chemoprophylaxis, and institutional outbreak management: clinical practice guidelines of the Infectious Diseases Society of America. Clin Infect Dis. 2009:48:1003-32. doi:10.1086/598513.

3. Süß T, Remschmidt C, Schink S, Luchtenberg M, Haas W, Krause G, Buchholz U. Facemasks and intensified hand hygiene in a German household trial during the 2009/2010 influenza a (H1N1) pandemic: adherence and tolerability in children and adults. Epidemiol Infect. 2011;139(12):1895-901. doi:10.1017/S0950268810003006.

4. Tracht SM, Del Valle SY, Hyman JM. Mathematical modeling of the effectiveness of facemasks in reducing the spread of novel influenza a (H1N1). PLoS One. 2010;5(2):e9018. doi:10.1371/journal.pone.0009018.

5. World Health Organization. Advice on the use of masks in the community setting in Influenza A(H1N1) outbreaks. 2009. Retrieved from http://www. who.int/csr/resources/publications/Adviceusemaskscommunityrevised.pdf

6. Tang CSK, Wong CY. Psychosocial factors influencing the practice of preventive behaviors against the severe acute respiratory syndrome among older Chinese in Hong Kong. J Aging Health. 2005;17:490-506. doi:10.1177/ 0898264305277966.

7. Nexøe J, Kragstrup J, Søgaard J. Decision on influenza vaccination among the elderly: a questionnaire study based on the health belief model and the multidimensional locus of control theory. Scand J Prim Health Care. 1999;17:105-10.

8. Biddle SJH, Hagger MS, Chatzisarantis NLD, Lippke S. Theoretical frameworks in exercise psychology. In: Tenenbaum G, Eklund RC, editors. Handbook of sport psychology. 3rd ed. New York, NY: Wiley; 2007. p. 537-59.

9. Brewer NT, Chapman GB, Gibbons FX, Gerrard M, McCaul KD, Weinstein ND. Meta-analysis of the relationship between risk perception and health behavior: the example of vaccination. Health Psychol. 2007;26:136-45. doi:10.1037/0278-6133.26.2.136. 
10. Godin G, Vézina-Im LA, Naccache H. Determinants of influenza vaccination among healthcare workers. Infect Control Hosp Epidemiol. 2010;31:689-93. doi:10.1086/653614.

11. Ajzen I. From intentions to actions: a theory of planned behavior. In: Kuhl J, Beckmann J, editors. From intentions to actions: a theory of planned behavior. Berlin: Springer; 1985. p. 11-39.

12. McEachan RRC, Conner M, Taylor NJ, Lawton RJ. Prospective prediction of health-related behaviours with the theory of planned behaviour: a metaanalysis. Health Psychol Rev. 2011;5:97-144. doi:10.1080/17437199.2010. 521684.

13. Rich A, Brandes K, Mullan BA, Hagger MS. Theory of planned behavior and adherence in chronic illness: a meta-analysis. J Behav Med. 2015;38:673-88. doi:10.1007/s10865-015-9644-3.

14. Gallagher S, Povey R. Determinants of older adults' intentions to vaccinate against influenza: a theoretical application. J Public Health. 2006;28:139-44. doi:10.1093/pubmed/fdl008.

15. Liao Q, Cowling BJ, Lam WWT, Fielding R. Factors affecting intention to receive and self-reported receipt of 2009 pandemic (H1N1) vaccine in Hong Kong: a longitudinal study. PLoS One. 2011;6(3):e17713. doi:10.1371/journal. pone.0017713.

16. Deci EL, Ryan RM. Intrinsic motivation and self-determination in human behavior. New York: Plenum; 1985.

17. Deci EL, Ryan RM. The "what" and "why" of goal pursuits: human needs and the self-determination of behavior. Psychol Inq. 2000;11:227-68. doi:10.1207/ S15327965PLI1104_01.

18. Williams GC, Grow VM, Freedman ZR, Ryan RM, Deci EL. Motivational predictors of weight loss and weight-loss maintenance. J Pers Soc Psychol. 1996;70:115-26.

19. Su YL, Reeve J. A meta-analysis of the effectiveness of intervention programs designed to support autonomy. Educ Psychol Rev. 2011;23:159-88. doi:10.1007/s10648-010-9142-7.

20. Chan DKC, Yang SX, Mullan B, Du X, Zhang X, Chatzisarantis NL, Hagger MS. Preventing the spread of H1N1 influenza infection during a pandemic: autonomy-supportive advice versus controlling instruction. J Behav Med. 2015;38:416-26. doi:10.1007/s10865-014-9616-z.

21. Turner KW. Senior citizens centers: what they offer, who participates, and what they gain. J Gerontol Soc Work. 2004;43:37-47. doi:10.1300/ J083v43n01_04.

22. Hagger MS, Chatzisarantis NLD. Integrating the theory of planned behaviour and self-determination theory in health behaviour: a meta-analysis. $\mathrm{Br}$ J Health Psychol. 2009;14:275-302.

23. Hagger MS, Chatzisarantis NLD. An integrated behavior-change model for physical activity. Exerc Sport Sci Rev. 2014;42:62-9. doi:10.1249/JES. 0000000000000008

24. Chan DKC, Fung YK, Xing S, Hagger MS. Myopia prevention, near work, and visual acuity of college students: integrating the theory of planned behavior and self-determination theory. J Behav Med. 2014;73(3):369-80. doi:10.1007/ s10865-013-9494-9.

25. Chan DKC, Hagger MS. Autonomous forms of motivation underpinning injury prevention and rehabilitation among police officers: an application of the trans-contextual model. Motiv Emot. 2012;36(3):349-64. doi:10.1007/ s1 1031-011-9247-4.

26. Chan DKC, Hagger MS. Theoretical integration and the psychology of sport injury prevention. Sport Med. 2012;42:725-32. doi:10.1007/BF03262291.

27. Hagger MS, Trost N, Keech J, Chan DK, Hamilton K. Predicting sugar consumption: application of an integrated dual-process, dual-phase model. Appetite. 2017;116(1):147-56. doi:10.1016/j.appet.2017.04.032.

28. Hagger MS, Chatzisarantis NLD, Barkoukis V, Wang CKJ, Baranowski J. Perceived autonomy support in physical education and leisure-time physical activity: a cross-cultural evaluation of the trans-contextual model. J Educ Psychol. 2005;97:376-90. doi:10.1037/0022-0663.97.3.376.

29. Hagger MS, Lonsdale AJ, Hein V, Koka A, Lintunen T, Pasi H, et al. Predicting alcohol consumption and binge drinking in company employees: an application of planned behaviour and self-determination theories. $\mathrm{Br}$ J Health Psychol. 2012;17:379-407. doi:10.1111/j.2044-8287.2011.02043.x.

30. Rhodes RE, de Bruijn GJ. How big is the physical activity intention-behaviour gap? A meta-analysis using the action control framework. Br J Health Psychol. 2013;18:296-309. doi:10.1111/bjhp.12032.

31. Sniehotta FF, Presseau J, Araújo-Soares V. Time to retire the theory of planned behaviour. Health Psychol Rev. 2014;8:1-7. doi:10.1080/17437199. 2013.869710.
32. Sniehotta FF, Scholz U, Schwarzer R. Bridging the intention-behaviour gap: planning, self-efficacy, and action control in the adoption and maintenance of physical exercise. Psychol Health. 2005;20:143-60. doi:10.1080/ 08870440512331317670

33. Levesque CS, Williams GC, Elliot D, Pickering MA, Bodenhamer B, Finley PJ. Validating the theoretical structure of the treatment self-regulation questionnaire (TSRQ) across three different health behaviors. Health Educ Res. 2007;22:691-702. doi:10.1093/Her/Cyl148.

34. Ajzen I. Constructing a TPB questionnaire: conceptual and methodological considerations. 2002. Retrieved from http://people.umass.edu/aizen/tpb.html

35. Muthén LK, Muthén BO. Mplus user's guide. 7th ed. Los Angeles, CA: Muthén \& Muthén; 1998-2012.

36. Marsh HW, Hau KT, Grayson D. Goodness of fit evaluation in structural equation modeling. In: Maydeu-Olivares A, McArdle J, editors, Psychometrics. A Festschrift for Roderick P. McDonald. Hillsdale, NJ: Erlbaum, 2005. p. 275-340.

37. Yu CY. Evaluating cutoff criteria of model fit indices for latent variable models with binary and continuous outcomes. University of California, Los Angeles: Unpublished doctoral dissertation; 2002.

38. Ng JY, Ntoumanis N, Thøgersen-Ntoumani C, Deci EL, Ryan RM, Duda JL, Williams GC. Self-determination theory applied to health contexts a metaanalysis. Perspect Psychol Sci. 2012;7:325-40. doi:10.1177/ 1745691612447309.

39. Fishbein $M$, Ajzen I. Predicting and changing behavior: the reasoned action approach. New York, NY: Psychology Press; 2009

40. Noar SM, Head KJ. Mind the gap: bringing our theories in line with the empirical data - a response to commentaries. Health Psychol Rev. 2014;8: 65-9. doi:10.1080/17437199.2013.855593.

41. Bandura A. Social foundations of thought and action: a social-cognitive theory. Englewood Cliffs, NJ: Prentice-Hall; 1986

42. Schwarzer R. Modeling health behavior change: how to predict and modify the adoption and maintenance of health behaviors. Appl Psychol. 2008;57: 1-29. doi:10.1111/j.1464-0597.2007.00325.x.

43. Hagger MS, Chatzisarantis NLD. The trans-contextual model of autonomous motivation in education: conceptual and empirical issues and meta-analysis. Rev Educ Res. 2016;86:360-407. doi:10.3102/0034654315585005.

44. Orbell S, Sheeran P. 'Inclined abstainers': a problem for predicting health related behaviour. Br J Soc Psychol. 1998;37:151-65. doi:10.1111/j.2044-8309. 1998.tb01162x

45. Conner M, Sheeran P, Norman P, Armitage C. Temporal stability as a moderator of relationships in the theory of planned behaviour. $\mathrm{Br} \mathrm{J}$ Soc Psychol. 2000;39:469-93. doi:10.1348/014466600164598.

46. Li KK, Chan DKS. Goal conflict and the moderating effects of intention stability in intention-behavior relations: physical activity among Hong Kong Chinese. J Sport Exerc Psychol. 2008;30:39-55.

47. Sheeran P, Abraham C. Mediator Of moderators: temporal stability of intention and the intention-behavior relation. Personal Soc Psychol Bull. 2003:29:205-15. doi:10.1177/0146167202239046.

48. Heckhausen $\mathrm{H}$, Gollwitzer PM. Thought contents and cognitive functioning in motivational and volitional states of mind. Motiv Emot. 1987;11:101-20. doi:10.1007/BF00992338

49. Gardner B. A review and analysis of the use of 'habit' in understanding, predicting and influencing health-related behaviour. Health Psychol Rev. 2015:9:277-95. doi:10.1080/17437199.2013.876238.

50. Keatley DA, Clarke DD, Hagger MS. Investigating the predictive validity of implicit and explicit measures of motivation on condom use, physical activity, and healthy eating. Psychol Health. 2012;27:550-69. doi:10.1080/ 08870446.2011 .605451

51. Rebar AL, Loftus AM, Hagger MS. Cognitive control and the non-conscious regulation of health behavior. Front Hum Neurosci. 2015;9:122. doi:10.3389/ fnhum.2015.00122.

52. Labrecque J, Wood W. What measures of habit strength to use? Comment on Gardner (2015). Health Psychol Rev. 2015;9:303-10. doi:10.1080/ 17437199.2014.992030.

53. Hagger MS, Chan DKC, Protogerou C, Chatzisarantis NLD. Using metaanalytic path analysis to test theoretical predictions in health behavior: an illustration based on meta-analyses of the theory of planned behavior. Prev Med. 2016:89:154-61. doi:10.1016/j.ypmed.2016.05.020

54. Cole DA, Preacher KJ. Manifest variable path analysis: potentially serious and misleading consequences due to uncorrected measurement error. Psychol Methods. 2014;19:300-15. doi:10.1037/a0033805. 